\title{
Gestión educativa para la sostenibilidad académica en Colombia
}

\author{
Lorenzo Portocarrero-Sierra $^{{ }^{*}}$ Jorge A. Restrepo-Morales ${ }^{1}$ Marisol Valencia-Cárdenas ${ }^{1}$ y Linda K. Calderón-Vera ${ }^{2}$ \\ (1) Facultad de Ciencias Administrativas y Económicas del Tecnológico de Antioquia I.U., CII 78b \#72 ${ }^{\mathrm{a}}$-220 Robledo, \\ Medellín, Colombia. Grupo de investigación RED. Correo-e: loposi@yahoo.es; jrestrepo@tdea.edu.co; \\ mvalenci1@tdea.edu.co \\ (2) Facultad de Educación y Ciencias Sociales del Tecnológico de Antioquia I.U., Cll 78b \#72ª-220 Robledo, Medellín, \\ Colombia. Grupo de investigación OBSERVATOS. Correo-e: lindacv1127@gmail.com
}

* Autor a quien debe ser dirigida la correspondencia.

Recibido Mar. 9, 2021; Aceptado May. 12, 2021; Versión final Jun. 9, 2021, Publicado Oct. 2021

\begin{abstract}
Resumen
El principal objetivo de este estudio es explicar la relación entre la gestión y la sostenibilidad de las instituciones de educación superior públicas (IESPs) y determinar los aspectos relevantes a intervenir en pro de la sostenibilidad y de la facilitación de la toma de decisiones. Se definen asociaciones entre factores latentes: economía, eficiencia, eficacia y calidad. Se recurre a un modelo de ecuaciones estructurales con una base de datos que recopila las percepciones de rectores y vicerrectores de 42 IESPs de Colombia en el año 2017. Los resultados exhiben la relación entre gestión y sostenibilidad y muestran que el indicador de la calidad depende de la investigación. El indicador de economía es influenciado de forma significativa por el presupuesto en infraestructura, hardware y software, seguido de inversión en personal y en planta docente. Se concluye que la eficacia depende tanto de la satisfacción de empleados como docentes, así como de la evolución salarial de los egresados.
\end{abstract}

Palabras clave: gestión educativa; calidad; educación superior; modelos multivariados

\section{Educational management for academic sustainability in Colombia}

\begin{abstract}
This primary aim of the present study is to explain the relationship between management and sustainability of public higher education institutions (IESPs, in Spanish) and to determine the aspects relevant for intervention to favor sustainability and to facilitate decision-making. Associations are defined based on the following latent factors: economics, efficiency, efficacy, and quality. A structural equations model is applied to a database that collects perception of rectors and vice-chancellors from 42 Colombian IESPs. The results reveal the relationship between management and sustainability and show that the quality indicator depends on research. The economy indicator is significantly influenced by budget for infrastructure, hardware, and software, followed by investment on staff and teaching staff. It is concluded that efficiency depends on the satisfaction of employees, including professors, and depends on the salary trends of IESPs graduates.
\end{abstract}

Keywords: education management; quality; efficiency; higher education; multivariate models 


\section{INTRODUCCIÓN}

Reformas educativas en los últimos años han llevado al desarrollo de nuevos modelos organizacionales para las entidades de educación superior (Moreno, 2017). Por esto, los países destinan gran parte de sus recursos a favorecer el crecimiento académico de los sistemas públicos de educación con énfasis en la prestación de un servicio con altos estándares de calidad. Para el caso colombiano, la Educación Superior es un servicio público que materializan las Instituciones de Educación Superior Públicas -IESP-; las cuales deben cumplir determinados criterios económicos, logísticos y académicos para ofertar programas, que están determinados por el Sistema de Aseguramiento de la Calidad en Educación Superior - Saces_, igualmente, por su propia voluntad, se pueden someter al proceso de acreditación institucional de alta calidad a través del Consejo Nacional de Acreditación - CNA-, que exige rigurosamente varios criterios entre los que se ubican las prácticas de buen gobierno, la planeación estratégica, los planes de mejoramiento, y los recursos financieros, entre otros (CNA, 2015).

Estas características dan cuenta de la importancia de una gestión eficiente que garantice niveles altos de calidad tanto institucional, como de programas académicos, situación que exige a los rectores y directivos de las IESP, orientar su ejercicio hacia la estrategia, haciendo uso de la combinación entre la gerencia con la política pública; sin embargo, ésta aun presenta falencias que ameritan revisarse (Broucker et al., 2018). Sin embargo, es responsabilidad de los rectores de las IESP alcanzar estos estándares rigurosos en alta calidad, aun, cuando presentan un desafío con respecto a los recursos que perciben desde el ente nacional, que generalmente no son suficientes para cubrir sus necesidades institucionales. Por tal motivo, se presentan grandes retos para la gestión pública y para la sostenibilidad de las instituciones que conforman el sistema de educación superior público de Colombia; una vez que la forma de financiación actual no es flexible y no alcanza a sostener las tasas de crecimiento respecto del acceso, equidad, calidad y pertinencia, afirma el Consejo Nacional de Educación Superior - CESU— (2014).

De acuerdo con disposiciones legales y normativas, entre las que se incluye la Ley 715 (2001), la cual establece los criterios para la distribución de recursos, cuyas fuentes de financiación se asignan con base en tasas de cobertura, calidad, reporte de información, planes de fomento a la educación superior, entre otras. En este orden de ideas, se plantea una investigación que explica la relación entre la calidad de la gestión educativa y la sostenibilidad de las IESP en Colombia, con el propósito de determinar los aspectos más relevantes de la gestión que podrían revisarse, vigilarse, facilitando la toma de decisiones del sistema y su sostenibilidad y facilitar la toma de decisiones en éste sentido, con fundamento en la información obtenida de la percepción de la alta dirección (rectores) de las organizaciones educativas; así como de los indicadores examinados respecto a eficacia, eficiencia, economía y calidad de gestión; que permitan orientar el sistema de educación superior pública en Colombia más allá de las políticas de gobierno y, centrarse en políticas de Estado; es decir, que no dependa de la constante transición y cambios gubernamentales. De lo que subyace aspectos como: la planeación de corto y largo plazo, la organización estructurada de los esquemas de financiación y la coherencia en los lineamientos normativos; lo que propicia condiciones de permanencia, acceso y calidad.

Las IESP se ven enfrentadas a responder por altas exigencias de gestión, aun cuando los recursos con que cuentan son limitados para la cobertura, que permita cumplir objetivos misionales de docencia, investigación y extensión con proyección social, convirtiéndose en un factor limitante de la gestión, que amerita requerimientos de control y vigilancia, procesos que son de necesario seguimiento especialmente durante crisis (Martínez-Campillo y Fernández-Santos, 2020). Las organizaciones del Estado, han transitado hacia la llamada 'Nueva Gestión Pública' —NGP_ cuya orientación está dada a la estrategia antes que a los procesos y cuyo interés principal consiste en la optimización de recursos y la posibilidad de medirse a través de indicadores que faciliten la toma de decisiones en la institución, en favor del logro de los objetivos misionales con eficacia, eficiencia, economía y calidad $(3 E+C)$.

En ese sentido, se plantean los desafíos frente a la sostenibilidad, que han mostrado transformaciones hacia modelos de negocio que se encuentran en proceso de elaboración, pero que conservan esquemas híbridos de financiación, o incluso, de capital mixto (Weerawardena et al., 2021). Entre las ventajas que se deducen de diversos modelos de negocio, se encuentra que las empresas que tienen propósitos asociados con financiamiento de mercado, eligen voluntariamente mejores niveles de transparencia financiera, el buen uso de los fondos, la transparencia financiera, mejorando el papel del estado en sus modelos de gobernanza (Goodell et al., 2020). El propósito del presente estudio es explicar la relación entre la gestión y la sostenibilidad de las IESP y determinar los aspectos relevantes a intervenir en pro de la sostenibilidad y de facilitar la toma de decisiones.

En correspondencia, la carencia de un esquema financiero eficiente en función de la sostenibilidad a partir de dimensiones de gestión pública en las IESP, es la prioridad en materia de educación superior en Colombia. Dichas dimensiones subyacen en las acciones para el uso de los recursos que permitan encontrar eficiencia, 
eficacia y calidad, aspectos que intervienen en cuanto a la administración, investigación y formación, lo que procura directamente la sostenibilidad de las mismas, facilitando resultados y aportes para la definición de la política pública en materia de distribución de recursos estatales para la educación superior.

Mediante esta investigación se propone explicar la incidencia de las dimensiones de economía, eficiencia, eficacia en la calidad de la gestión educativa para la sostenibilidad de las instituciones de educación superior oficiales en Colombia usando un modelo de Ecuaciones Estructurales, luego de aplicar un cuestionario a rectores y vicerrectores de una muestra representativa de las Instituciones de Educación Superior Públicas del país. Lo anterior, desde la gestión pública se podrían identificar aspectos de las organizaciones educativas que faciliten la optimización de los recursos disponibles y provean la supervivencia de éstas instituciones en el marco de la calidad de la educación superior que enuncia el Ministerio de Educación Nacional -MEN-, con el ánimo de realizar aportes a la gestión pública de estas organizaciones educativas, así como el manejo eficiente de los recursos, que permitan el cumplimiento de las exigencias de calidad, enmarcadas en el funcionamiento normal y la ejecución de los objetivos misionales.

Para el análisis de los indicadores de gestión que evidencian la eficacia, eficiencia, economía y calidad (3E + C) de las IESP, se consideran las diversas variables que se han encontrado en relación a la eficiencia y la calidad académica de Martínez-Campillo y Fernández-Santos(2020) sobre gestión pública estratégica, las combinaciones de capital intelectual y nuevos modelos pedagógicos, que favorecen la innovación y los entrenamientos en los centros educativos, generando mejor calidad académica (Yami et al., 2021).

El análisis para determinar la correlación entre el desempeño de universidades estatales de México con los indicadores de excelencia académica y el nivel de asignación de recursos financieros con origen en fuentes de financiamiento del gobierno federal (Pantoja-Aguilar, 2019), el desenvolvimiento económico de Schumpeter (1963). Además, el nuevo paradigma que encamina los modelos sin ánimo de lucro a una transformación basada en innovación de modelos de negocio que consideran esquemas híbridos de financiación (Weerawardena et al., 2021), aspectos que se traducen en posibilidades de obtener una sostenibilidad financiera como aspecto clave en la función organizacional de entidades sin ánimo de lucro como las IESP. Lo anterior, facilita conservar o expandir los servicios de educación en la oferta académica, mientras se desarrolla la resiliencia a las luchas económicas ocasionales en el corto plazo.

\section{OTROS ANTECEDENTES}

Este estudio se desarrolla a la luz del modelo de gerencia pública, para ello se hace preciso explicitar como se entiende este modelo de gestión. La gerencia pública surge como una propuesta administrativa gubernamental que pretende modernizar los enfoques de la alta dirección del Estado con el propósito de perfeccionar la capacidad gubernamental para mejorar la gobernabilidad e implementar el concepto del mejoramiento continua en los procesos administrativos, para resolver de forma eficiente y eficaz los retos presentes en la sociedad. Es así, como algunas de las variables asociadas con la gestión y la calidad de la educación consideran los resultados derivados de la inversión, como la infraestructura, la contratación docente, o los servicios sociales, médicos, administrativos brindados a la comunidad; otras variables pueden involucrar la creación de nuevo conocimiento, procesos de investigación, donde tenga inherencia algún proceso de formación o participación estudiantil. Es así como la descripción de áreas latentes, o también denominadas, dimensiones, debe partir de un constructo teórico que se relaciona con cada una de su significado intrínseco. Las dimensiones a considerar son: Calidad de la gestión educativa, Economía, Eficiencia y Eficacia. Dichas dimensiones latentes harán parte de la estimación de los Modelos de Ecuaciones Estructurales, con el fin de determinar y caracterizar el tipo de asociación que exista entre las variables estimadas con otras observables.

\section{Dimensión calidad de la gestión educativa}

La calidad educativa puede considerar diversos aspectos que, en suma, buscan resultados óptimos, tanto para los egresados, como para las Instituciones de Educación Superior. Calvo (2018), asocia la calidad con inversión institucional, dadas las necesidades del mercado laboral y los planes de desarrollo gubernamentales. En tanto que Portocarrero-Sierra et al. (2020), analizan cuáles son las dimensiones de corte social, económico, financiero y de gestión, que diferencian de forma correcta a las instituciones de educación superior públicas en Colombia acreditadas de las que no tienen tal reconocimiento. Desde otra óptica, Melo et al. (2017), la relaciona con procesos pedagógicos, de manera que las universidades establezcan relaciones también con otras organizaciones para planificar el proceso formativo; por ejemplo, las necesidades del entorno se pueden transformar para que las instituciones se acerquen más a lo que requiere el medio, desarrollando acciones como aumentar incentivos para la investigación y becas, así como estrategias de formación intrínsecas con apoyo de empresas, para aumentar la empleabilidad.

El desempeño laboral puede fungir como indicador del grado en que la universidad, por medio de sus procesos de enseñanza-aprendizaje, genere competencias en los estudiantes para resolver el encargo social de cada 
programa ofertado; para ello, se exige a la misma, medirse y autoevaluarse para mantener planes de mejoramiento y mantenimiento que faciliten el alcance de su objetivo. En la misma línea, Steur et al. (2012) plantean que, si bien la graduación estudiantil tiene relevancia, también la tiene el desempeño profesional, por ello es importante evaluar la capacidad de las instituciones para realizar el entrenamiento encaminado a la empleabilidad de los egresados. Adicionalmente, algunas de las necesidades de las empresas de hoy implican el análisis de las transformaciones digitales para la optimización de sus estrategias, que van desde el aumento de investigación, el mejoramiento tecnológico y ajuste de capacidades (Büyüközkan y Göçer, 2018), implicando una nueva mirada de la educación en el ámbito de personal empresarial y de profesionales en formación.

Por su parte, las competencias investigativas de los egresados permiten desarrollar habilidades para el desarrollo de proyectos y propuestas de investigación, así como la generación de conocimiento científico en su contexto, y, por ende, mejor desempeño académico y profesional, lo cual los hace más propositivos y les da valor como profesionales (González et al., 2019; George y Salado, 2019). En la misma línea, Böttcher y Thiel (2018), desarrollaron un enfoque innovador para modelar las competencias de investigación en diferentes disciplinas académicas mediante un modelo de competencias que denominaron el modelo RMRC$\mathrm{K}$, el cual considero cinco dimensiones: habilidades para revisar el estado de la investigación, habilidades metodológicas, habilidades para reflexionar sobre los resultados de la investigación, habilidades de comunicación y conocimiento del contenido, concluyendo como los estudiantes se podrían beneficiar a futuro, tanto como investigadores junior o futuros profesionales, del desarrollo de competencias investigativas en su proceso de formación profesional de cualquier disciplina.

Para ello las Instituciones de Educación Superior requieren invertir presupuesto, tener altas capacidades en los docentes orientadores, adecuada planeación de programas académicos, óptimo servicio a los estudiantes, entre otros aspectos necesarios para que los estudiantes cumplan a cabalidad con los requerimientos exigidos para obtener un título académico, que lo acredita como un profesional idóneo que la sociedad necesita. Calvo (2018) sostiene que el país no ha contado con un alto nivel de recursos invertidos en investigación, como consecuencia se presenta un desarrollo incipiente de dichos niveles, lo cual induce poca participación de estudiantes.

Igualmente, la calidad también se refleja en las capacidades para el logro de resultados. Al respecto es importante el desarrollo de competencias asociadas al manejo de TICS, como buscar, obtener, evaluar y administrar información para hacer una transformación al conocimiento. Adicionalmente, se requieren habilidades para organizar y analizar la información a partir de las múltiples fuentes para deducir los nuevos conocimientos. Lo anterior, demanda apoyos digitales y de trabajo colaborativo con grupos de investigación para compartir búsquedas y análisis en red con altas competencias tecnológicas, así como socializar los hallazgos o resultados de la investigación por medio de publicaciones o eventos (George y Salado, 2019).

Otro factor importante es el servicio proporcionado por la institución, para responder de manera directa y rápida a las necesidades de los usuarios; aquí se consideran factores como oportunidad, accesibilidad, precisión y continuidad en la entrega de los servicios; es así como la calidad se puede perfeccionar en la medida en que se mejoren los atributos o particularidades de los servicios entregados. En Colombia, el Consejo Nacional de Acreditación —CNA—, determina los criterios a evaluar frente a la calidad de la Educación Superior en el país, sobre los cuales recomienda al Ministerio de Educación Nacional -MEN-, la certificación de alta calidad a las IESP que logran alcanzarlos. No obstante, estos factores no están planteados en términos de metas y cifras concretas.

En ese sentido, Pedraja et al. (2020), indican como la calidad se refiere a la capacidad institucional para responder de manera directa y rápida a las necesidades de los usuarios. Además de lo relacionado con el desempeño profesional, la calidad del egresado se asocia con la incorporación de competencias blandas como, la actitud, los valores, el trabajo en equipo, capacidad de aprendizaje, ética, entre otras; algunas de las cuales dependen del nivel de profundización en el currículo (Steur et al., 2012). La OCDE enfatiza en definir unos indicadores universales para ser utilizados por todos los países. Así, los indicadores tendrían que medir características permanentes, perdurables de los sistemas de educación para que sea posible su análisis longitudinal; por ello deben ser fácilmente inteligibles por audiencias amplias y describir situaciones actuales a la vez que facilitan las comparaciones educativas.

En esa línea, Delahoz et al. (2020), subrayan la existencia de evidencia empírica que señala la relación entre la acreditación de calidad y el buen desempeño de los estudiantes en pruebas estandarizadas, además determinan que la dimensión de comunicación escrita, considerada una competencia blanda, es un factor crítico para determinar la acreditación de universidades en el sistema educativo colombiano. Así mismo, la acreditación institucional es claramente otra manera de realizar la medición acerca de la calidad educativa, teniendo en cuenta muchos aspectos y factores institucionales, siendo otra mirada de los resultados generalizados como indicador de resultados. En este trabajo, la calidad se aprecia desde una mirada alterna, asumiendo que parte desde la percepción de los directivos, como medida producida por el trabajo investigativo 
de los docentes, grupos y estudiantes que se conforman, logrando colaborativamente productos de nuevo conocimiento, innovación o formación del talento humano, lo cual se traduce en una clasificación de grupos que cumple con los estándares de medición nacionales, como también se consideran aspectos de empleabilidad y el crecimiento percibido en resultados de las pruebas de estado Saber Pro.

\section{Dimensión economía}

La economía es la capacidad de las instituciones para movilizar recursos en favor del cumplimiento de los objetivos, de manera eficiente, aspectos que son responsabilidad de los gerentes (Portocarrero-Sierra et al., 2020). En instituciones públicas, el autor propone que se consideran varios aspectos relacionados con la economía, como: capacidad de autofinanciamiento, ejecución presupuestal acorde a lo planeado, así como capacidad de recuperación de préstamos. Los recursos económicos son parte fundamental para un apropiado funcionamiento de las entidades de Educación Superior, puesto que de un óptimo manejo se derivan docentes de calidad y satisfechos laboralmente, así como los buenos resultados de los estudiantes.

Uno de los aspectos discutidos acerca de la economía en educación superior atañe a óptimos resultados con bajo nivel de recursos invertidos, sin embargo, se presenta divergencia desde el punto de vista conceptual de diversos autores. Es entonces importante la revisión de los aspectos relevantes para el estudio de la economía y la eficiencia, como lo explica Velasco (2008), cuando determina la demanda de educación Superior a partir del precio de la matrícula, del ingreso de los hogares, niveles de renta y la pérdida de otros bienes. Igualmente, define una función de producción educativa, utilizando como variables de entrada (inputs) la cantidad de equipos, las características de edificios, la cantidad de docentes y alumnos, así como características personales y socioeconómicas, y como resultados, por ejemplo, las pruebas de conocimiento, aptitudes y destrezas matemáticas, habilidades, actitudes y valores. Sin embargo, Velasco concluye que no todos los autores confirman la tesis de que, a mayor inversión de recursos, hay mejores resultados en los alumnos, no obstante, también señala que algunos autores relacionan el aumento de recursos, con un manejo eficiente, generando mejores resultados en los conocimientos de los alumnos.

Otros trabajos evalúan los resultados en materia educativa con respecto a los recursos invertidos, es el caso de Ramírez et al. (2019) quienes luego de realizar una revisión documental, con el enfoque metodológico Knowledge Development Process - Constructivist (Proknow-c), indican que la mayor parte de los autores no acepta medir resultados sin considerar la inversión para obtenerlos. Teixeira et al. (2017) muestran que las Instituciones de Educación superior públicas y privadas requieren de la gestión de indicadores financieros para un funcionamiento adecuado. En una línea muy similar se ajusta Williams (2016), señalando, además, que una población que en mayor cantidad accedan a una muy buena educación superior tendrán mejor nivel económico a nivel local.

Adicionalmente, muestran que la eficiencia educativa se puede determinar por la cantidad de resultados de las IES, pero su existencia y fidelidad depende en gran medida de la forma de administración. En la misma línea, Zámečník y Výstupová (2014) afirman que, al verificar los rankings de las universidades, no es clara una relación significativa de estos con la eficiencia en educación superior, lo cual abre brechas de investigación en este sentido. Por último, se resalta la tesis de Hedges et al. (1994) citada en Velasco (2008, Pág. 86) quienes usando el "meta-análisis», "concluyen que el gasto por alumno sí que incide en los resultados de los alumnos. En general, HEDGES y sus colaboradores hallan una correlación positiva entre inputs o recursos escolares y resultados educativos para todos los inputs", aprobando el uso de la función de producción o características similares, como se aborda en esta investigación, considerando el presupuesto invertido en diferentes variables asociadas con la Educación Superior.

\section{Dimensión eficiencia}

La eficiencia, relaciona un conjunto de resultados con cierto nivel de recursos, en educación, se puede interpretar como un resultado operativo derivado del trabajo de la comunidad educativa que tiene unas entradas o recursos para producir unas salidas o resultados. La eficiencia indica la capacidad de optimizar los recursos, sin embargo, esta medida en educación superior no sigue un mismo estándar. Muchos trabajos se centran en medir la eficiencia de las instituciones de educación superior con base en el rendimiento de estudiantes, sin embargo, otras mediciones se focalizan en factores institucionales como planta docente, formas de ingreso, becas, o nivel de ingresos (Melo et al. 2017).

Una alternativa para medir la eficiencia es la técnica de fronteras eficientes, valoración estadística, que depende de un conjunto de variables de salida y otras de entrada, estableciendo una asociación entre éstas por medio de una estimación óptima. En el caso de lbáñez et al. (2017), encuentran que la tasa de graduación por carrera universitaria, como indicador de eficiencia, está influenciada por el perfil de los alumnos y las condiciones del entorno, hallando además que su incremento es producido por la cantidad de becas ofertadas, además de una mejor formación docente, la residencia de los alumnos, colegio y cupos por programa, así como la realización de procesos de investigación, resaltando que oferta educativa y la cantidad de becas son aspectos que deberían ser mejorados en las IES para aumentar el desempeño y tasa de graduación de estudiantes. 
El presupuesto de las universidades es diferente según su categoría, pública o privada, por ende, requiere de una planeación adecuada para el logro de eficiencia en el manejo de los recursos. Una de las mayores dificultades que afrontan las Universidades para lograr adecuada calidad académica, es la inversión, por ello, los incrementos realizados en las matrículas para Instituciones con capital mixto o Universidades Privadas, son una de los principales insumos para el fortalecimiento de la investigación, capacidad docente, e infraestructura, sin embargo, requiere muy buen manejo de los recursos recibidos, con lo anterior también soportado en la expansión de programas ofertados, para lo cual debe ser eficiente de forma financiera y productiva.

Melo et al. (2017) muestran un estudio de eficiencia bajo una función de producción estimada con los criterios de desempeño académico de los estudiantes, con relación a variables de entrada como son aspectos institucionales y socioeconómicos, encontrando un impacto positivo en la eficiencia, cuando se ven incrementos en variables como infraestructura, personal docente, además, con el aumento del ingreso de los padres y mejor entorno de vida. Por medio de esos efectos de las variables institucionales, se pueden vincular el mejoramiento de infraestructura y personal docente con el aumento de programas, debido a que estos lo exigen para una mejor oferta académica, al mismo tiempo que incrementan los ingresos por sus matrículas; así que el logro de un incremento en la cobertura puede significar los incrementos de capacidad docente, infraestructura, resultados investigativos y mejoramiento del desempeño del profesional.

Lo anterior se une a la relación entre graduados y cobertura, vinculado al indicador del total de estudiantes que terminan con respecto al total que iniciaron cada cohorte, sin embargo, esta medida puede variar por los cambios de programa de los estudiantes, reingresos, convenios, entre otros aspectos. La eficiencia medida desde las áreas de cobertura implica que la entidad oferte más cupos con garantías y servicios que permitan llevar el mayor número posible a la graduación, debido a que aumentarían variables como desempeño y empleabilidad, mejorando el nivel de eficiencia en la entidad educativa, aspecto que se convierte en una hipótesis de este trabajo.

\section{Dimensión eficacia}

La Eficacia en este trabajo se asume como el cumplimiento de resultados óptimos de la misión educativa, lo cual se manifiesta en una satisfacción de la comunidad con la institución, además del logro de ingresos adecuados para los egresados, una buena empleabilidad, como resultado de la pertinencia de los programas para responder a las necesidades del medio. La eficacia converge en un mejoramiento de las capacidades de desempeño, alineadas con las necesidades locales o regionales que, a su vez, se combinan con los planes de desarrollo. Adicional a lo anterior, se debe revisar el posicionamiento de la institución, su reconocimiento facilita el incremento de inscripciones y matrículas, así como el desempeño de los estudiantes y el crecimiento de empleabilidad, lo cual repercute en satisfacción con las condiciones de formación y, por ende, incrementa la credibilidad y la sostenibilidad de la entidad educativa. A nivel financiero, lo anterior se traduce en que el buen logro de resultados bajo un buen manejo de los recursos, lleva a la permanencia de las IES y fidelidad de estudiantes acorde con Ramírez et al. (2019). En esta medida, la eficacia posee una connotación de crecimiento en empleabilidad de sus egresados que, además, promueve continuidad en la labor educativa para aumentar la satisfacción de sus empleados y estudiantes, facilitando su existencia y permanencia.

\section{Modelos de Ecuaciones Estructurales -MES-}

Algunos modelos estadísticos permiten analizar efectos de variables asociadas con las instituciones, o la inversión, los factores asociados al servicio, la atención o el bienestar dado a los estudiantes sobre la calidad del egresado. Uno de los esquemas de modelación, es el MES que basa sus estimaciones en una estructura paramétrica a partir de un conjunto de variables latentes definidas como dimensiones, que son construidas a partir de variables medibles u observables, con las cuales se establece el análisis del factor confirmatorio (AFC). Por ello, a partir de una base con variables observables, se estiman las variables latentes o factores usando su matriz de correlaciones, analizando las relaciones de dependencia entre las variables involucradas, así como modelos de regresión entre las mismas latentes (Kyriazos, 2018), lo cual facilita estimar el tipo de efecto entre la latente exógena y la endógena que juega el papel de la respuesta o resultado, por ello, sus aplicaciones son transversales en especial, para ciencias sociales, humanas o administrativas.

Diferentes MES han sido empleados para evaluar, por ejemplo, la calidad educativa, o relacionar el promedio del estudiante, con algunas pruebas de desempeño en su carrera. Cabana et al. (2016), establecen constructos como los indicadores de calidad del estudiante medido a través de la percepción de satisfacción y autoevaluación de aprendizaje, encontrando que cuando el estudiante percibe mayor calidad también encuentra mayor satisfacción y fidelización; similarmente, la red de apoyo externo se encontró asociada con la consecución de prácticas, trabajo independiente, lo cual impacta adecuadamente la fidelidad del estudiante. En este sentido, los autores encuentran que el apoyo de la institución y el docente son factores positivos frente a la calidad percibida por el estudiante (Cabana et al., 2016).

Otro modelo MES fue estimado luego de aplicar un cuestionario a 647 estudiantes universitarios chinos, para evaluación de características de eficiencia académica, así como la calidad de desarrollo de estos en el 
proceso educativo, aquí se encuentra que otros aspectos relacionados con dicha calidad son el grado de participación del estudiante y la capacidad de construcción, es decir, sus propios desarrollos juegan papeles importantes en los procesos de enseñanza-aprendizaje y calidad educativa (Liu, et al., 2014). Los autores encuentran que los estudiantes que más se involucran o participan, y que además reciben incentivos, incrementan los efectos positivos de la eficiencia académica. Una síntesis de los hallazgos y relaciones temáticas se presenta en la figura 1.

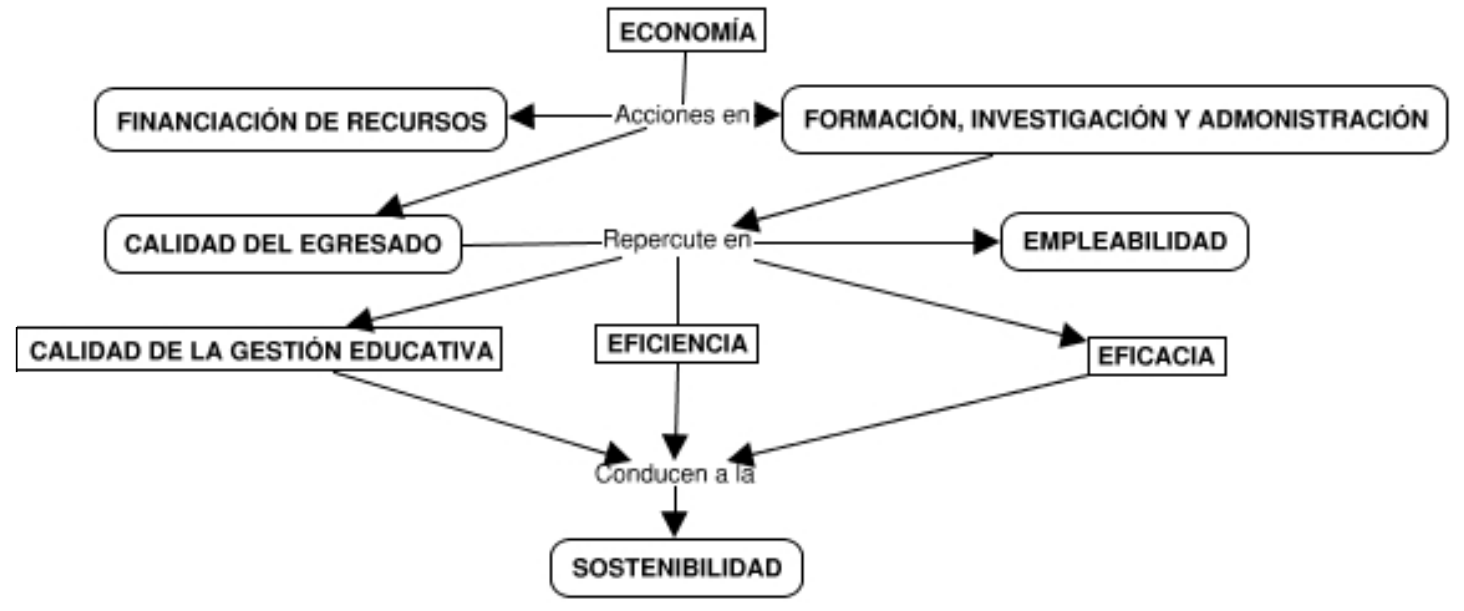

Fig. 1: mapa conceptual de los hallazgos y relaciones temáticas.

\section{METODOLOGÍA}

La metodología de investigación es de tipo cuantitativa con alcance explicativo y analítico; la población de estudio fueron 61 instituciones de educación superior públicas existentes a corte 31 de diciembre de 2017, las cuales se identificaron de acuerdo a su carácter (universidad, institución universitaria o escuela tecnológica, institución tecnológica o institución técnica profesional), de acuerdo a su naturaleza jurídica (nacional, departamental o municipal) y a su ubicación en el territorio nacional; se aplicó un instrumento de encuesta a rectores y vicerrectores de Entidades de Educación Superior Públicas del país, lo cual muestra que es información de fuentes primarias, tienen alta credibilidad y validez.

Se contactaron rectores y vicerrectores de diferentes instituciones de educación superior públicas (IESP), sin recibir retribuciones por las respuestas dadas. De un total de 81 IESP, se excluyeron 19 de carácter especial, ya que corresponden a un presupuesto diferente, quedando 62 IES como población de referencia, de estas responden 42 ( $68 \%$ del total), la cual es estimada usando un nivel de confianza estadístico del $95 \%$ y un nivel de error del $8.5 \%$, lo cual garantiza un acierto en los cálculos que se generen a partir de los datos recolectados. Las preguntas fueron enviadas por medio electrónico en un cuestionario digital, sistematizando las respuestas con el fin de realizar el análisis usando la percepción de los expertos sobre las variables observadas y su consistencia.

A partir de la información obtenida del instrumento, las variables se midieron de la siguiente forma: La variable dependiente fue a través del Índice de Sostenibilidad financiera = ISF = EBITDA/Activo Total (AT), de las variables independientes, la eficiencia se midió a través del Indicador = inversión por estudiante, la economía se midió a través del indicador de margen operacional y la calidad se midió a través de si contaba o no con Acreditación Institucional.

Se precisa que en la validez del instrumento se considera la validez de contenido a través de 5 expertos toda vez que el constructo obedece a las áreas del conocimiento de administración, educación y metodología. Las respuestas son independientes, puesto que es una sola respuesta por institución y no hay conocimiento entre los expertos que responden el cuestionario. Adicionalmente se realizan las pruebas de KMO, Alfa de Cron Bach, que determinan la consistencia de la base de datos recolectada, indicando que el nivel de correlación entre variables, mas no entre individuos, es adecuado para el análisis SEM.

Para medir el grado de dependencia de cada variable con ella misma, se aplicó la prueba de auto correlación de Box Pierce a la matriz de datos transformada por medio de una raíz cuadrada (la escala continua) y se realizó un conteo de casos en que cada variable muestra un valor p mayor de $5 \%$, indicando que son independientes. Dicho total se divide entre el total de variables de la base de datos usados en su cálculo; su resultado muestra que los datos no presentan sesgos o correlación serial, pues el $92.9 \%$ de las variables muestran tener independencia, siendo éste un porcentaje que representa la mayoría de variables de la base de datos. Lo anterior indica que no hay sesgos en la base de datos. 
Un primer acercamiento a la comprensión de los modelos MES, puede ser a partir del estudio de los diagramas relacionales por medio de los cuales pueden proponerse, o estimarse, el efecto posible causado entre variables usando flechas que se direccionan de una a la otra. Para ello también es importante establecer unos constructos definidos como dimensiones que se definen como variables latentes, estimados por medio de variables medibles u observables. El Modelo de Ecuaciones Estructurales (MES), busca establecer un conjunto de asociaciones entre factores latentes, que en este caso corresponden a las dimensiones: economía, eficiencia, eficacia y calidad. Por medio de dichos análisis, se establece qué tan significativo puede ser el aporte individual o no, de las variables medidas y las latentes y entre éstas últimas. La función entre las variables observadas y las latentes por medio del Análisis del Factor Confirmatorio, pueden ser descritas por medio de la ecuación (1).

$$
\xi_{\mathrm{i}}=\mathrm{f}\left(\mathrm{X}_{\mathrm{j}}, \theta\right)
$$

Ejemplo: i) $\xi_{1}$ Variable latente denominada dimensión de Economía; ii) $\xi_{2}$ Variable latente denominada como dimensión Eficacia; iii) $\xi_{3}$ Variable latente denominada como dimensión Eficiencia; iv) $\eta_{1}$ Variable latente de resultado definida como la dimensión de Calidad de la Gestión Educativa. Para realizar el proceso de estimación en este trabajo, se usa el paquete Lavaan, con la función sem del programa $R$ un software estadístico gratuito.

\section{Componente técnico de los Modelos de Ecuaciones Estructurales}

En los MES se deben establecer las dimensiones que hipotéticamente podrían tener relación de dependencia o efectos, por ello, se proponen unas variables observadas por cada una de dichas dimensiones, por parte del equipo investigador; lo anterior permite estimarlas con su estructura de varianza-covarianza con el Análisis del Factor Confirmatorio (AFC); seguido a esto, el modelo facilita que por medio de las variables latentes estimadas, se construya el análisis de regresión y el análisis de covarianzas, definiendo cuáles son las variables latentes de tipo respuesta (Endógena) y de tipo covariable (Exógena), con dicha matriz (Baena et al., 2014; Liu et al., 2014). Los modelos MES permiten una comprensión cuantitativa sobre las relaciones causales que conectan los conjuntos de variables, que podrían ser representadas, por ejemplo, por medio de diagramas tipo path o relacionales (Escobedo et al., 2016), lo cual facilita determinar cómo se dirigen las asociaciones entre las áreas por analizar.

El diagrama causal o Path podría tener por ejemplo, una variable endógena $\left(\eta_{1}\right)$, variable no observada pero constituida por otras medibles, dos variables exógenas que pueden denominarse $\xi 1$ y $\xi 2$, y ser también estimadas por otras variables medibles, como se aprecia en la Figura 2, con las denominaciones $\lambda_{\mathrm{ij}}$, las $\xi_{\mathrm{i}}$ son variables latentes exógenas o independientes, que pueden tener o no asociación con la variable latente endógena o dependiente que puede ser más relevante, $\eta_{1}$, la cual podría percibir los efectos de las otras latentes; $y$ los valores $\gamma_{i j}$ miden los efectos que pueden asociar dichas variables no tangibles.

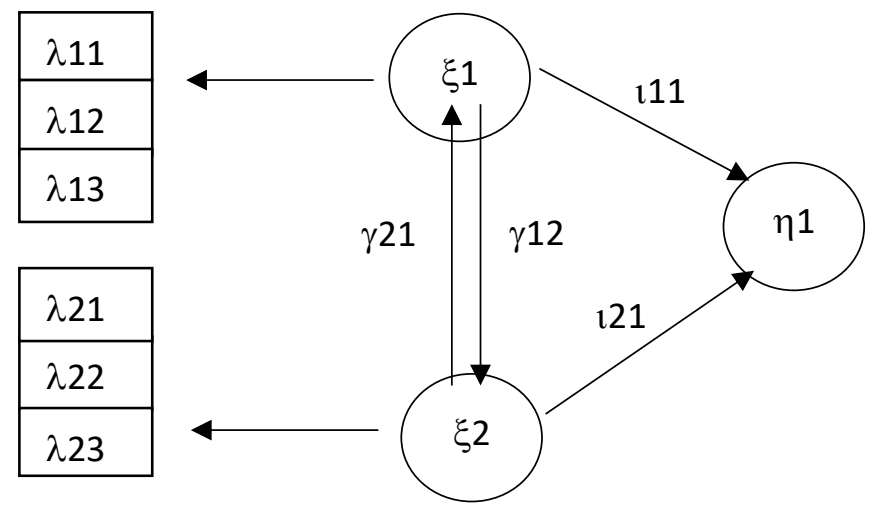

Fig. 2. Ejemplo de Diagrama de relaciones causales. (Adaptada de Escobedo et al., 2016)

\section{Componentes de ajuste de los modelos de ecuaciones estructurales}

El modelo MES usa teoría paramétrica y la estructura de varianza-covarianzas y correlación entre las variables observables, indicando los efectos que producen éstas sobre las latentes y efectos de latentes entre sí. Además, cuenta con indicadores que permiten estimar el nivel de adecuación o consistencia del modelo estimado, como el índice de comparación de ajuste (CIF-Comparative Fit Index), sugiriendo a menudo que, para encontrar cálculos de modelos aceptables, éste debe tener un valor superior al $90 \%$. La Raíz del Error Cuadrático Medio (RMSEA), a menudo se sugiere que tenga valores inferiores a $6 \%$, aunque en algunos casos, bajo validación de los modelos para encontrarlos aceptables pueden tener valores cercanos al $8 \%$. Los modelos MES no siempre cumplen con las condiciones que implican valores cercanos a 1 para los 
indicadores CFI, TLI, NFI, por ello es posible comparar varios modelos usando también el RMSEA (Olmedo et al. 2017).

Baena et al. (2014) afirman que es posible usar la prueba de significancia del modelo con base en la estadística chi-cuadrado, el GFI (índice de bondad de ajuste), que indica la proporción de varianza explicada por el modelo, el NFI (el índice de ajuste normalizado), el CFI (el índice de ajuste comparativo), y el RMSE (Error de aproximación cuadrático medio). Los índices NFI y CFI, que varían de 0 a 1 , el mejor ajuste se da con valores cercanos a 1, pero el NFI es sensible al tamaño de muestra, por lo cual se prefiere el CFI (Camanzi et al. 2018), por su parte, se prefieren valores pequeños del RMSEA o rangos que se presenten entre $0.05 \mathrm{y}$ 0.08. Por otro lado, antes de realizar las estimaciones, es posible verificar la consistencia del instrumento y la base de datos usada, con alfa de Cronbach, así como comprobar la validez de alguno de los resultados a partir de otro modelo estadístico.

\section{RESULTADOS}

Las estimaciones del modelo de Ecuaciones Estructurales son realizadas en el software R. En primer lugar, se muestra el valor de la prueba del alfa de Cronbach, para mostrar la consistencia del modelo estimado. La prueba del grado de relación conjunta, es de $68 \%$, indicando una consistencia aceptable para el conjunto de variables usadas para las estimaciones del análisis del factor. Kaiser-Meyer-Olkin Statistics - Llamado: KMOS ( $\mathrm{x}=$ prueba $\mathrm{km}$ )- Resultado: KMO-Criterio: $69 \%$. El alfa de Cronbach estimado con las variables de la base de datos usada en las estimaciones, tiene un valor de $93 \%$, mostrando que la base tiene una buena asociación y correlación, esto implica que la base de datos usada es consistente para la estimación propuesta. La tabla 1 muestra los indicadores del modelo SEM estimado, generan confianza ya que se presenta una explicación de variabilidad aproximado del $95 \%$ en el índice CFI, para los resultados mostrados a continuación.

Tabla 1. Indicadores de ajuste del modelo SEM.

\begin{tabular}{|l|c|c|}
\hline & Estándar & Robusto \\
\hline Modelo de prueba de usuario: & & \\
\hline Estadístico de Prueba & 2329,066 & 1170,894 \\
\hline Grados de Libertad & 734 & 734 \\
\hline P-valor (Chi-cuadrado) & 0 & 0 \\
\hline Factor de corrección de escala & & 4,027 \\
\hline Estadístico de Prueba & 31102,042 & 6182,96 \\
\hline Grados de Libertad & 780 & 780 \\
\hline P-valor & 0 & 0 \\
\hline $\begin{array}{l}\text { Modelo de usuario versus modelo de } \\
\text { línea de base: }\end{array}$ & & \\
\hline Índice de ajuste comparativo (CFI) & 0,947 & 0,919 \\
\hline Índice de Tucker-Lewis (TLI) & 0,944 & 0,914 \\
\hline RMSEA & 0,230 & 0,120 \\
\hline $\begin{array}{l}\text { Intervalo de confianza del 90 por ciento } \\
\text { - inferior }\end{array}$ & 0,220 & 0,107 \\
\hline $\begin{array}{l}\text { Intervalo de confianza del 90 por ciento } \\
\text { - superior }\end{array}$ & 0,241 & 0,133 \\
\hline P-valor RMSEA <= 0,05 & 0,000 & 0,000 \\
\hline
\end{tabular}

La estimación del Análisis de Factor Confirmatorio para obtener cada uno de los factores o variables latentes, muestra los valores $p$ de cada una de las variables observables inferiores al $5 \%$, que es un nivel de error adecuado; lo anterior indica que la estimación es conveniente, presentando un buen grado de asociación y cohesión de las variables observables para estimar las latentes. La dimensión de calidad de gestión educativa, se observa ponderada por aspectos de los egresados como la empleabilidad, su continuidad en posgrados, también por los resultados de las pruebas de estado Saber Pro, así como una variable sobre resultados del crecimiento en investigación. El indicador de Economía refleja que es influenciado por el presupuesto invertido en diversos campos de forma significativa, por ejemplo, el efecto estimado es muy alto para los recursos en infraestructura, hardware y software, seguido de inversión en personal y en docentes, reflejando la dirección principal de dicho indicador. Ahora bien, la eficacia se ve ponderada principalmente por la satisfacción de empleados y docentes, así como el crecimiento salarial de los egresados. 
La tabla 2 muestra la estimación de los modelos de regresión de la estructura multivariada, entre las covariables y variable respuesta latente, aspectos que indican que la calidad de la gestión educativa sí recibe influencias significativas de las demás latentes construidas por medio del modelo SEM: Economía, Eficacia y Eficiencia, las cuales, al mostrarse con efectos positivos (Estimación), muestran que se producen aumentos en las percepciones sobre de ésta. Lo anterior indica que la calidad de la gestión educativa, cuyas connotaciones se relacionan con desempeño académico y empleabilidad de egresados, además, de los aportes investigativos acorde con la percepción de los rectores, es incrementada gracias a los efectos de la economía, eficiencia y eficacia en el manejo de recursos y aspectos.

Tabla 2. Modelos de regresión de las variables latentes

\begin{tabular}{|l|r|r|r|r|}
\hline \multicolumn{1}{|c|}{ Regresiones: } & \multicolumn{1}{c|}{ Estimación } & $\begin{array}{c}\text { Error } \\
\text { Estándar }\end{array}$ & \multicolumn{1}{c|}{ z-valor } & $\mathrm{P}(>|\mathrm{z}|)$ \\
\hline Calidad & & & & \\
\hline Economía & 0,118 & 0,102 & 1,148 & 0,251 \\
\hline Eficacia & 0,121 & 0,046 & 2,626 & 0,009 \\
\hline Eficiencia & 0,546 & 0,162 & 3,369 & 0,001 \\
\hline
\end{tabular}

\section{DISCUSIÓN Y ANÁLISIS}

En Colombia el reto para los rectores de las IESP, como servidores públicos adquiere dimensiones mayores cada día, en primer lugar porque el nuevo enfoque de la gestión pública impone un cambio de paradigma, para que se conviertan en gerentes públicos y formulen propuestas que den respuesta a los problemas circunstanciales de la educación, para ello es imperativo replantear los procesos y procedimientos para dirigirlos en una lógica de competencia y derribar prácticas insanas de la administración pública como son: ausencia de rendición de cuentas, la corrupción y el gasto excesivo, entre otros. Para ello la calidad, como un constructo teórico que circunscribe múltiples dimensiones (Pantoja-Aguilar,2019), emerge como un factor administrativo que requiere mantener un cauce fluido entre sus dimensiones: calidad de estudiantes y profesores, los procesos de organización, gestión y bienestar estudiantil y profesoral porque estos factores juegan un rol determinante para mejorar la confianza de la ciudadanía hacia los funcionarios públicos; en ese sentido, los hallazgos frente a la calidad académica evaluada en este documento, evidencia la incidencia positiva de procesos investigativos y académicos, como elementos centrales de la función académica y la estructura organizacional de las IES. Lo anterior, da respuesta a Estrada, F. M. (2011), quien propone la necesidad de enfocar la atención hacia procesos de certificación que soporten el diseño de un modelo de gestión de calidad aplicable a las administraciones públicas latinoamericanas.

Otro aspecto relevante es la inversión de recursos en diferentes aspectos; donde desde la dimensión económica, se encuentra que altos valores de inversión en la infraestructura, el hardware y software, tienen relación positiva y significativa en la calidad de la educación. Estos hallazgos son avalados por González et al. (2019), quienes afirman que el uso de herramientas informáticas apoyan a las IES y sus estudiantes, en la selección de actividades para fomentar habilidades centrado en sus intereses, estilos de aprendizaje, aptitudes, preferencias y por tanto, se mejoran los resultados de aprendizaje; cuya proxis de medición en este trabajo son los resultados de las pruebas de Saber Pro, donde se encuentra una asocian positiva entre el aumento en las puntuaciones y la mejor percepción de calidad académica. Se resalta la incidencia positiva de la calidad dependiendo del crecimiento de cobertura en relación con los estudiantes admitidos y los programas académicos, en correspondencia con las necesidades del mercado laboral (Yami et al., 2021).

En la misma línea, Delahoz et al. (2020), ratifican como los procesos administrativos y operativos propuestos de un modelo gestión de alta calidad tienen impacto directo en el desempeño de las pruebas de estado; lo que es corroborado en este estudio donde se reafirma que aumentar la cantidad de grupos de investigación y la cantidad de investigadores en las categorías más altas, así como el crecimiento en resultados de las pruebas de Saber Pro, y de la cantidad de egresados con empleo, se traducen en mayores índices de calidad. Así como, las medidas de crecimiento en empleabilidad, la satisfacción del personal y de los docentes, son aspectos que indican y revelan que los factores institucionales tienen fuertes asociaciones con el buen desempeño de los estudiantes, por ende, de los profesionales, traduciéndose en que el buen manejo de los recursos puede reflejar eficiencia productiva acorde con el objetivo educativo.

En concordancia con el hallazgo de que los gastos de inversión en personal administrativo, docente y de funcionamiento, inciden positivamente en la calidad de la gestión educativa, además, también el incremento de la calificación en la satisfacción de los docentes, y empleados. Estos hallazgos, son congruentes con Pedraja et al. (2020), quienes afirman la necesidad de contar con líderes activos y la promoción de culturas eficaces, por su potencial de influir en el cumplimiento de los objetivos organizacionales y en el logro de la calidad institucional, afectando así la formación de profesionales competentes. 
Por último, la tasa de deserción incide de forma negativa en el indicador de eficacia y, por ende, se afecta la calidad. Dichos aspectos son además asociados con respecto a la empleabilidad, ya que una percepción en crecimiento de los salarios de enganche de los egresados se traduce en incrementos indirectos en la calidad de los egresados. Lo anterior resalta también, que la percepción de los directivos de las IESP muestra la importancia de un adecuado manejo Eficiente, Eficaz y Económico de los recursos para encontrar mejores resultados educativos, y empleabilidad, además de que proveen un consenso acerca del efecto de la investigación y los esfuerzos en la clasificación sobre la valoración de la calidad de los resultados educativos.

\section{CONCLUSIONES}

La investigación realizada genera resultados importantes para el análisis del nuevo modelo de gestión púbica de las Instituciones de Educación Superior Públicas, ya que se resaltan aspectos que no competen a las condiciones personales de los estudiantes, sino que se relacionan con factores institucionales, como la gestión óptima de recursos, inversión en infraestructura, software, satisfacción docente, y la inversión en investigación; además del crecimiento de programas y cobertura educativa, ya que la calidad de la gestión educativa se ve favorecida por dichos factores indiscutiblemente.

Desde el punto de vista teórico, se corrobora como el nuevo enfoque de la gestión pública trae consigo un cambio de paradigma, que exige a los servidores públicos repensar su rol de gerentes públicos para pasar de actores pasivos a tornarse en gestores del cambio mediante la formulación de estrategias que mitiguen los problemas casuales del sistema educativo colombiano. De forma paralela, se requiere políticas públicas para modificar los procesos y procedimientos de medición de la calidad de la educación para enfocarlos bajo principios de competitividad para derrumbar vicios latentes en la administración pública como la corrupción y el gasto excesivo, entre otros.

Los resultados corroboran como la calidad incluye es un concepto multi dimensional que exige sincronizar diferentes factores como estudiantes, profesores, egresados, empleados, sector privado y comunidad en general para optimizar la confianza de los diferentes actores hacia la administración pública focalizando esfuerzos en modelos de gestión congruentes con las administraciones de recursos públicos en Latinoamérica.

Se resalta que las percepciones de los rectores y vicerrectores tienen alto grado de consenso. El manejo de los recursos en las Entidades de Educación Superior, dadas las inversiones en infraestructura, software, capacitación docente e investigación, surte efectos en el crecimiento educativo, desarrollo, empleabilidad de los egresados, lo cual se verifica a través de las acciones de los rectores y vicerrectores de las Instituciones de Educación Superior Públicas del país, acorde y ratificados con los resultados de esta investigación. La limitación de este estudio es que se tomó información de la percepción de los rectores de las IESP, por lo tanto, puede variar la precisión en las respuestas, por el hecho que los encuestados tienen intereses particulares en cada institución.

\section{REFERENCIAS}

Baena, A., Granero A., Sánchez J., y Martínez, M., Modelo Predictivo de la Importancia y Utilidad de la Educación Física, Cuadernos de Psicologia del Deporte, 14(2), 121-30 (2014)

Böttcher, F., y Thiel, F., Evaluating Research-Oriented Teaching: A New Instrument to Assess University Students' Research Competences, https://doi.org/10.1007/s10734-017-0128-y, Higher Education, 75(1), 91-110 (2018)

Broucker, B., De Wit, K., y Verhoeven, J. Higher Education for Public Value: Taking the Debate Beyond New Public Management, http://dx.doi.org/10.1080/07294360.2017.1370441, Higher Education Research \& Development, 37(2) 227-240 (2018)

Büyüközkan, G., y Göçer, F., Digital Supply Chain: Literature Review and a Proposed Framework for Future Research, https://doi.org/10.1016/j.compind.2018.02.010, Computers in Industry, 97, 157-177 (2018)

Cabana, R., Cortés, F., Vega, D., y Cortés. R., Análisis de la Fidelización del Estudiante de Ingeniería con su Centro de Educación Superior: Desafíos de Gestión Educacional, http://dx.doi.org/10.4067/s0718-50062016000600009, Formación Universitaria, 9(6),93-104 (2016)

Calvo, J., Calidad Educativa en la Educación Superior Colombiana: una Aproximación Teórica, DOI: http://dx.doi.org/10.18634/sophiaj.14v.2i.799, Sophia-Educación, 14(2),4-14 (2018)

Camanzi, L., Arba, E., y otros tres autores, A Structural Equation Modeling Analysis of Relational Governance and Economic Performance in Agri-Food Supply Chains: Evidence from the Dairy Sheep Industry in Sardinia, https://doi.org/10.1186/s40100-018-0099-z, Agricultural and Food Economics, 6(1) (2018)

Delahoz, E., Guillen, S., y Fontalvo, T., Análisis de la Acreditación de Calidad en Programas de Ingeniería Industrial y los Resultados en las Pruebas Nacionales Estandarizadas en Colombia, http://dx.doi.org/10.4067/S0718-

50062020000100127, Formación Universitaria, 13(1), 127-134, (2020) 
Escobedo, M., Hernández, J., Ortega, V., y Martínez, G., Modelos de Ecuaciones Estructurales: Características, Fases, Construcción, Aplicación y Resultados, http://dx.doi.org/10.4067/s0718-24492016000100004, Ciencia y Trabajo, 18(55), 16-22 (2016)

George, C., y Salado, L., Competencias Investigativas con el uso de las TIC en Estudiantes de Doctorado, https://doi.org/10.32870/ap.v11n1.1387, Apertura, 11(1) 40-55 (2019)

González, J., Carrillo, A., y otros cuatro autores, Runayaysoft: Adaptive System for Supporting the Selection of Enrichment Activities in Educative Environments, https://doi.org/10.1108/ijwis-04-2018-0021, International Journal Of Web Information Systems, 15(1), 103-131, (2019)

Goodell J.W., Goyal A., y Hasan, I., Comparing Financial Transparency Between For-Profit and Nonprofit Suppliers of Public Goods: Evidence From Microfinance, Journal of International Financial Markets, Institutions \& Money, 64. 101146, (2020)

Ibáñez, M., Morresi, S., y Delbianco, F., Una Medición de Eficiencia Interna en una Universidad Argentina usando el Método de Fronteras Estocásticas, https://doi.org/10.1016/j.resu.2017.06.002, Revista de la Educación Superior, 46(183), 47-62 (2017)

Kyriazos, T., Applied Psychometrics: Sample Size and Sample Power Considerations in Factor Analysis (EFA, CFA) and SEM in General, https://10.4236/psych.2018.98126, Psychology, 9(08), 2207-2230 (2018)

Liu, H., Liu, J., y Chi, X., Regulatory Mechanism of Self-Determination Involvement in Higher Education: Assessing Chinese Students' Experiences, https://doi.org/10.1007/s10734-013-9640-x, Higher Education, 67(1), 51-70 (2014)

Martínez-Campillo, A., y Fernández-Santos, Y., The Impact of the Economic Crisis on the (In)Efficiency of Public Higher Education Institutions in Southern Europe: the Case of Spanish Universities, https://doi.org/10.1016/j.seps.2019.100771, Socio-Economic Planning Sciences, 71, 100771 (2020)

Melo, L., Ramos, J., y Hernández, P., La Educación Superior en Colombia: Situación Actual y Análisis de Eficiencia, https://doi.org/10.13043/dys.78.2, Revista Desarrollo y Sociedad, (78), 59-111 (2017)

Moreno, C., Las Reformas en la Educación Superior Pública en México: Rupturas y Continuidades, https://doi.org/10.1016/j.resu.2017.03.001, Revista de la Educación Superior, 46(182). p 27-44 (2017)

Olmedo, E., Berrocal E., Olmos M., y Expósito, J., Structural Equations Model (SEM) of a Questionnaire on Evaluation of Intercultural Secondary Education Classrooms, https://doi.org/10.1016/s0121-4381(14)70013-x, Suma Psicológica, 21(2),107-115 (2014)

Pantoja-Aguilar, M., Indicadores de Desempeño Académico como Predictores de Captación de Recursos Financieros, https://doi.org/10.29101/crcs.v0i79.9222, Convergencia-Revista de Ciencias Sociales, 26(79), 01-27 (2019)

Pedraja, L., Marchioni, Í., Espinoza, C., y Muñoz, C., Liderazgo y Cultura Organizacional como Factores de Influencia en la Calidad Universitaria: Un Análisis Conceptual, http://dx.doi.org/10.4067/S0718-50062020000500003, Formación Universitaria, 13(5), 3-14, (2020)

Portocarrero-Sierra, L., Restrepo-Morales, J., y Arias-Calderón, J., Evaluación del Impacto de la Acreditación de Alta Calidad en las Instituciones Públicas de Educación Superior De Colombia. http://dx.doi.org/10.4067/S071850062020000600037, Formación Universitaria, 13(6), 37-50 (2020)

Ramírez, Z., Barrachina, M., y Ripoll, V., University Rankings Disclosure and Efficiency in Higher Education: A Bibliometric and Systematic Analysis, https://doi.org/10.4438/1988-592x-re-2019-384-412, Revista de Educacion, 2019(384), 255-286 (2019)

Steur, J., Jansen E., y Hofman W., Graduateness: An Empirical Examination of the Formative Function of University Education, https://doi.org/10.1007/s10734-012-9533-4, Higher Education 184(1), 89-104 (2012)

Velasco, M. Economía de la Educación: Aspectos Teóricos y Actividades Prácticas. $1^{\text {a }}$ ed., Pearson Prentice hall, Madrid, España, (2008)

Weerawardena J, Salunke S., Haigh N., y Sullivan G., Business model innovation in social purpose organizations: Conceptualizing dual social-economic value creation, Journal in Business Research, 125, 762-771 (2021)

Williams, G., Higher Education: Public Good or Private Commodity?, https://www.scienceopen.com/hosteddocument?doi=10.18546/LRE.14.1.12. London Review of Education, 14(1), 131-142 (2016)

Yami, S., M'Chirgui, Z., Spano, C., y Gontier-Barykina, O., Reinventing Science and Technology Entrepreneurship Education: The Role of Human and Social Capitals, https://doi.org/10.1016/j.techfore.2020.120044, Technological Forecasting and Social Change, 164 (120044). (2021)

Zámečník, R., y Výstupová, L., Consequences of Changes in Public Universities Funding on Applied Financial Management Tools, https://doi.org/10.1016/j.sbspro.2013.12.552, Procedia-Social and Behavioral Sciences, 109, 845$850(2014)$ 